\title{
Dynamically reinforced heterogeneous grain structure prolongs ductility in a medium-entropy alloy with gigapascal yield strength
}

\author{
Muxin Yang ${ }^{a, 1}$, Dingshun Yan ${ }^{a, 1}$, Fuping Yuan ${ }^{a, b}$, Ping Jiang ${ }^{a}$, Evan Ma ${ }^{c, 2}$, and Xiaolei $\mathbf{W u}^{\mathrm{a}, \mathrm{b}, 2}$

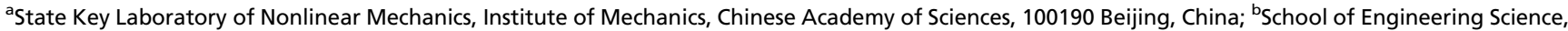 \\ University of Chinese Academy of Sciences, 100049 Beijing, China; and 'Department of Materials Science and Engineering, Johns Hopkins University, \\ Baltimore, MD 21218
}

Edited by William D. Nix, Stanford University, Stanford, CA, and approved June 4, 2018 (received for review May 6, 2018)

Ductility, i.e., uniform strain achievable in uniaxial tension, diminishes for materials with very high yield strength. Even for the CrCoNi medium-entropy alloy (MEA), which has a simple facecentered cubic (FCC) structure that would bode well for high ductility, the fine grains processed to achieve gigapascal strength exhaust the strain hardening ability such that, after yielding, the uniform tensile strain is as low as $\sim 2 \%$. Here we purposely deploy, in this MEA, a three-level heterogeneous grain structure (HGS) with grain sizes spanning the nanometer to micrometer range, imparting a high yield strength well in excess of $1 \mathrm{GPa}$. This heterogeneity results from this alloy's low stacking fault energy, which facilitates corner twins in recrystallization and stores deformation twins and stacking faults during tensile straining. After yielding, the elastoplastic transition through load transfer and strain partitioning among grains of different sizes leads to an upturn of the strain hardening rate, and, upon further tensile straining at room temperature, corner twins evolve into nanograins. This dynamically reinforced HGS leads to a sustainable strain hardening rate, a record-wide hysteresis loop in load-unload-reload stress-strain curve and hence high back stresses, and, consequently, a uniform tensile strain of $22 \%$. As such, this HGS achieves, in a singlephase FCC alloy, a strength-ductility combination that would normally require heterogeneous microstructures such as in dual-phase steels.

heterogeneous grain structure | ductility | medium-entropy alloy | back stress hardening

$\mathbf{T}^{\mathrm{h}}$ he equimolar multicomponent alloys, recently dubbed as the medium-entropy alloys (MEAs) (1-6) and high-entropy alloys (HEAs) (7-25), are emerging as an interesting class of structural materials. The single-phase $\mathrm{CrCoNi}$ and $\mathrm{FeCrMnCoNi}$ alloys of the face-centered cubic (FCC) structure, in particular, have exhibited a combination of high ultimate tensile strength, ductility, and fracture toughness $(1,2,5,9,10)$. However, their high tensile ductility is for micrometer-grained (MG) microstructure, for which the yield strength $\left(\sigma_{0.2}\right)$ is relatively low (below $\sim 400 \mathrm{MPa}$ at room temperature). When $\sigma_{0.2}$ approaches $1 \mathrm{GPa}$, achieved, for example, by refining the grain size, the common dilemma of strengthductility trade-off rears its ugly head $(3,7)$ : The stress-strain curve peaks immediately after yielding, exhibiting a drastic loss of ductility. The crux of the problem lies in the inadequate strain hardening, which can no longer keep up with the high flow stresses to circumvent plastic instability [localization of strain via necking, as predicted by the Considère criterion (26)]. This is, in fact, the norm for conventional metals when strengthened either by reducing their grain size or by cold working (27-29). For instance, $\sigma_{0.2}$ of a nanograined (NG) metal can be increased to many times that of its coarse-grained (CG) counterpart. However, such strengthening comes at the expense of strain hardening, because the intragranular dislocation storage upon straining, a potent mechanism for strain hardening, almost disappears (27). This is also true in ultrafine-grained (UFG) MEAs (3). Even though stacking faults (SF) are stored in large numbers due to the wellknown low SF energy (SFE) of these FCC HEAs/MEAs $(1,2,8$,
12), twinning became difficult in UFG grains, and, at room temperature, strain hardening remained inadequate to sustain uniform elongation after the MEA yields at $>1 \mathrm{GPa}$ (3). In general, plasticity-enhancing mechanisms in low-SFE alloys such as phase transformation [transformation-induced plasticity (TRIP)] (10, 30-33) and twinning [twinning-induced plasticity (TWIP)] (1, 2 , 34-41) all lose their potency in UFG and NG structures $(34,35$, 42, 43).

Here we advocate a strategy generally applicable for singlephase low-SFE alloys, to provide adequate strain hardening to delay necking. The CrCoNi MEA is a good model to work with, for setting an example for such alloys. The key idea underlying our microstructural design is as follows. We intentionally push for a highly heterogeneous grain structure (HGS) via partial recrystallization annealing following conventional cold rolling of CrCoNi (see Materials and Methods). This HGS, spanning NG, to UFG, to MG, provides a $>1-G P a$ yield strength similar to that of uniform UFG MEA (3). However, the more impactful consequence is that the HGS imparts load partitioning to promote internal stress hardening that produces an upturn in strain hardening rate, which is further aided by dynamically generated twinned NGs at the grain corners and grain boundaries along with tensile straining. These revived twins and NGs, not previously induced in large numbers at room temperature in uniform grains due to required high stresses, strengthen the alloy

\section{Significance}

Back stress hardening is usually not obvious in single-phase homogeneous grains, but can be made unusually large and sustained to large tensile strains by creating an unusually heterogeneous grain structure in single-phase alloys with low stacking fault energy (SFE), as demonstrated here for the facecentered cubic CrCoNi medium-entropy alloy. The low SFE facilitates the generation of twinned nanograins and stacking faults during tensile straining, dynamically reinforcing the heterogeneity. Large uniform tensile strain can be achieved after yielding even at gigapascal stress, in the absence of heterogeneities from any second phase.

Author contributions: X.W. designed research; E.M. and X.W. conceived the ideas; M.Y. carried out the mechanical property tests; D.Y. conducted EBSD observations; X.W. conducted TEM observations; M.Y., D.Y., F.Y., P.J., E.M., and X.W. contributed to data analysis and discussions; and E.M. and X.W. wrote the paper.

The authors declare no conflict of interest.

This article is a PNAS Direct Submission.

This open access article is distributed under Creative Commons Attribution-NonCommercialNoDerivatives License 4.0 (CC BY-NC-ND).

${ }^{1}$ M.Y. and D.Y. contributed equally to this work

${ }^{2}$ To whom correspondence may be addressed. Email: xlwu@imech.ac.cn or ema@jhu.edu. This article contains supporting information online at www.pnas.org/lookup/suppl/doi:10 1073/pnas.1807817115/-/DCSupplemental.

Published online June 26, 2018. 

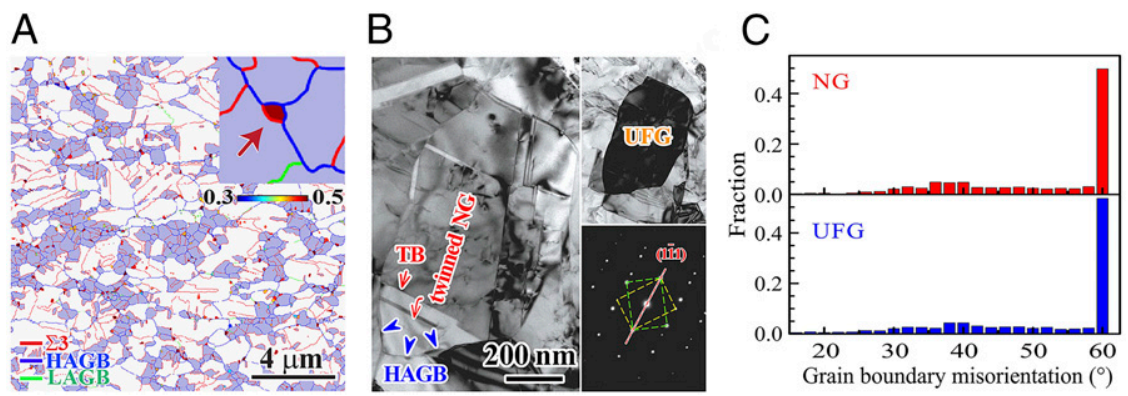

Fig. 1. HGS in CrCoNi MEA. (A) EBSD grain boundary image showing three grades of grain size: MG (white), micrometer-sized grains; UFG (light blue), ultrafine grains with submicron grain sizes; and NGs (red), nanograins with grain sizes below $250 \mathrm{~nm}$. NGs are colored based on the magnitude of the Schmid factor (see scale bar). (Inset) Close-up of a twinned NG (indicated by an arrow) nucleated at the TJ of UFGs. (B) TEM image showing a twinned NG at the lower part of a UFG. (Upper Inset) The entire UFG in (011) zone axis. (Lower Inset) Selected area electron diffraction pattern showing twin orientation relationship between the nanograin and parent UFG. (C) Distribution of grain boundary orientation for both UFGs and NGs in $A$. This HGS was produced via partial recrystallization annealing at $600{ }^{\circ} \mathrm{C}$ for $1 \mathrm{~h}$ following cold rolling of CrCoNi MEA to $95 \%$ thickness reduction.

and provide strain hardening to enable a large uniform tensile elongation at gigapascal flow stresses after yielding at $>1 \mathrm{GPa}$.

Fig. $1 A$ shows the high-angle grain boundaries (GBs) (HAGB, defined as those with misorientation of $\geq 15^{\circ}$ ) in the $\mathrm{CrCoNi}$ MEA after cold rolling and subsequent annealing at $600{ }^{\circ} \mathrm{C}$ for 1 h (see Materials and Methods), observed using electron backscatter diffraction (EBSD) imaging with a resolution of $40 \mathrm{~nm}$. The partial recrystallization resulted in a grain structure that is characterized by three levels of grains with obviously different sizes: MGs, UFGs, and NGs. The MGs (white) have a mean grain size of $2.3 \mu \mathrm{m}$, the intermediate UFGs (blue) range from $250 \mathrm{~nm}$ to $1 \mu \mathrm{m}$, and the smallest NGs are less than $250 \mathrm{~nm}$ (mostly in red, marked in terms of the magnitude of the Schmid factors for clear distinction from UFGs and MGs). The mean grain size of this HGS is $330 \mathrm{~nm}$. The number percentage of UFGs is $47 \%$, and that of NGs is $43 \%$. Interestingly, almost all NGs locate at the GBs and triple junctions (TJs) of UFGs; see an example in the close-up view in Fig. $1 A$, Inset. Such a recrystallization microstructure is expected for low SFE metals.

Fig. $1 B$ is a TEM image showing clearly a twinned NG locating at the lower part of its parent grain, apparently nucleated at the corner of the HAGB. Such a twinned grain is thus often called the corner twin $(44,45)$. In fact, this is a well-known mechanism of discontinuous nucleation for recrystallization, especially in metals of low SFE $(44,45)$. There are many annealing twins in MGs (Fig. $1 A$ ), as marked using red segments. Based on our EBSD analysis, both the NGs and UFGs are mostly twinned grains, judged based on twinning orientation relationship with their parent grains: Out of the HAGB (blue segment) with its parent grain, a significant portion is very often a $\Sigma 3$ twin boundary (TB, the red segment). Among the total NG or UFG grains, the percentage of twinned ones is as high as $72 \%$ and $43 \%$, respectively. This dominance of twin orientation is obvious in Fig. $1 C$.

Fig. $2 A$ displays the tensile engineering stress-strain $(\sigma-\varepsilon)$ curve of the HGS MEA tested at $298 \mathrm{~K}$. The alloy exhibits a $\sigma_{0.2}$ as high as $\sim 1,150 \mathrm{MPa}$, together with a tensile strain to failure of over $30 \%$. Note that the uniform elongation $\left(\mathrm{E}_{u}\right)$ is as large as $22 \%$. The $\sigma-\varepsilon$ curve preformed at $77 \mathrm{~K}$ is also shown, which indicates a simultaneous increase of $\sigma_{0.2}, \Theta$, and $\mathrm{E}_{u}$ with respect to roomtemperature values. Reproducible tests show clearly the consistent mechanical properties (SI Appendix, Fig. S1). Fig. 2 also compares the tensile response and strain hardening behavior with three other types of (much more homogeneous) grain structures. The first is the same CrCoNi MEA after high-pressure torsion and subsequent full recrystallization annealing (3), with a mean grain size (199 $\mathrm{nm})$ and yield strength not very different from our HGS (330 nm). Fig. $2 B$ shows the true flow stress $\sigma_{f}$ (minus $\sigma_{02}$ for a close-up view) versus true strain. The 199-nm MEA has only a $2 \% \mathrm{E}_{u}$ (curve 1). To increase $\mathrm{E}_{u}$ to $20 \%$ (to become comparable to our HGS, curve 2), ref. 3 had to increase the MEA grain size to restore strain hardening, and, as a consequence, dropped $\sigma_{0.2}$ by $\sim 330 \mathrm{MPa}$. In contrast, the HGS shows a transient hardening at strains ranging from 4 to $\sim 8 \%$, typical of strain hardening response in the initial stage in heterogeneous structures due to back stress hardening (46-49). Fig. $2 C$ shows the normalized strain hardening rate, $\Theta=1 / \sigma_{f} \cdot\left(\partial \sigma_{f} / \partial \varepsilon\right)$. Uniquely, the HGS shows an upturn of $\Theta$ within the range of the transient, and, more importantly, $\Theta$ is always higher than those of curves 1 and 2 throughout the entire tensile deformation. In contrast, the 199-nm MEA (curve 1) plunges straight to the onset of plastic instability predicted by the Considère criterion $(\Theta=1)$, very early on at a plastic strain of $<\sim 2 \%$. The $\Theta$ of curve 2 also shows a monotonic drop with strain, even though the stress level is $\sim 330 \mathrm{MPa}$ lower (3).

Next, we compare our HGS with two uniformly grained structures in 316L stainless steel (SS) of low SFE (50). Much like the comparison with more-uniform grained MEAs above, here again the SS having a $\sigma_{0.2}$ similar to HGS shows a very low $\mathrm{E}_{u}$ (curve 3 in Fig. $2 B$ ) and a plunging $\Theta$ (Fig. $2 C$ ), and lowering $\sigma_{0.2}$ by $\sim 330 \mathrm{MPa}$ only increased $\mathrm{E}_{u}$ to $\sim 10 \%$ (curve 4) (50), with a monotonic decreasing $\Theta$ (Fig. 2C).

However, another comparison is made in Fig. 2, with a conventionally-pure Ni, one that does not have low SFE. This Ni has a mean grain size of $500 \mathrm{~nm}$ (see SI Appendix, Fig. S2 for details), not too far away from the average in our HGS. Its yield strength is not too much lower either (Fig. $2 B$ ). However, as seen in Fig. $2 C$, its strain hardening rate is not sustainable (for its load/unload/reload tensile curve, see below). All these comparisons clearly demonstrate the advantage of HGS over those of much more homogeneous grains, regardless of their SFE, even when at a much-lowered $\sigma_{0.2}$. This highlights the crucial role played by our intentionally maximized HGS.

The resultant strength-ductility combination $\left(\sigma_{02}\right.$ and $\left.\mathrm{E}_{u}\right)$ is displayed in another format in Fig. 2D. First, comparing our HGS (red solid line) with the more uniform grained MEA (3) (dashed black line), $\mathrm{E}_{u}$ is much increased at any given $\sigma_{0.2}$. In fact, the higher the $\sigma_{0.2}$, the larger the relative increase in $\mathrm{E}_{u}$ (this difference is highlighted in Fig. $2 D$, Inset). This advantage of HGS is especially pronounced at high strengths toward $1 \mathrm{GPa}$. The second point to note in Fig. $2 D$ is that, although previous reports attempted to use phase transformation (10) or a combination of phase transformation and deformation twinning (51) to evade the trade-off between $\sigma_{02}$ and $\mathrm{E}_{u}$, they offer high $\mathrm{E}_{u}$ usually at low $\sigma_{0.2}(<\sim 400 \mathrm{MPa})$. More detailed comparisons are made in SI Appendix, Fig. S3.

The loading-unloading-reloading (LUR) tensile tests were conducted to unveil the underlying strain hardening mechanism and defect behaviors (Fig. 3A). Each LUR curve exhibits a hysteresis loop (Fig. 3B). Of special note is that the reverse (compressive) plastic flow sets in (at $0.2 \%$ offset strain) upon elastic unloading, even though the applied stress is still in tension (Fig. $3 B$ ). This is an unambiguous sign that unequivocally demonstrates the generation of high internal back stresses $(47-49,52)$. Several hysteresis loops in other metals $(47,53)$ known for strong back stress hardening are also included in the plot, for comparison. We have also made comparisons with two homogeneous microstructures. One is UFG Ni prepared via equal channel angular pressing followed by 

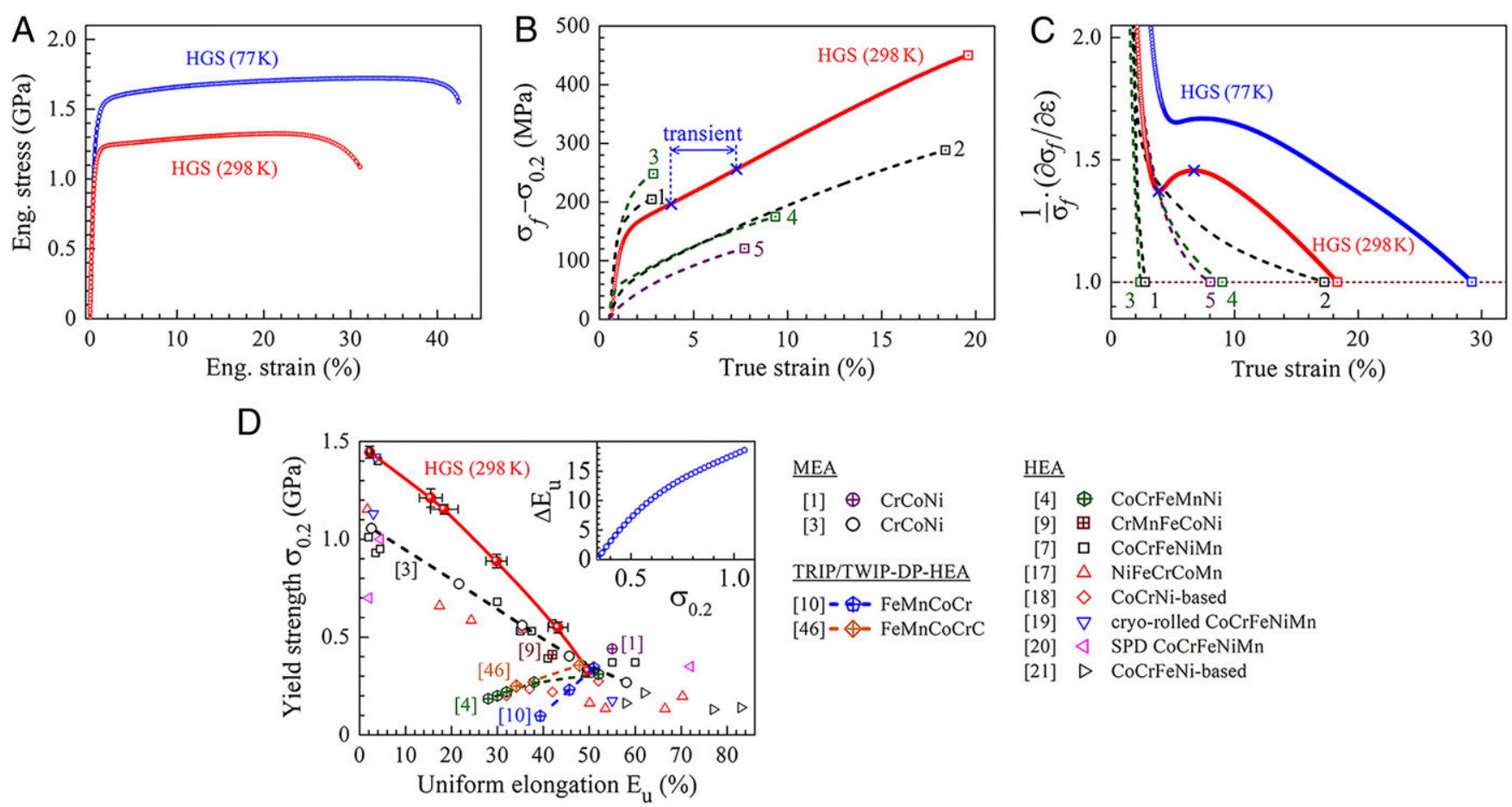

Fig. 2. Strain hardening and strength-ductility combination in HGS. ( $A$ ) Tensile engineering $\sigma-\varepsilon$ curve of HGS CrCoNi MEA at both 298 and $77 \mathrm{~K}$ after cold rolling and recrystallization annealing at $873 \mathrm{~K}$. (B) Flow true stress (minus $\sigma_{0.2}$ ) versus true strain curves. Note the presence of a transient hardening stage between the two inflection points (marked by $\times$ ) in the HGS curve. Five curves for much more homogenous grained structures are shown for comparison. Curves 1 and 2: 199-nm and 286-nm CrCoNi MEA (910 and $775 \mathrm{MPa}$ ) (3). Number in bracket: $\sigma_{0.2}$ (similarly hereinafter). Curves 3 and 4: 316 L SS (975 and $785 \mathrm{MPa}$, respectively) (50). Curve 5: UFG Ni (775 MPa) (see SI Appendix, Fig. S2 for details). (C) Normalized strain hardening rate ( $\Theta$ ) versus true strain curves. Note the upturn of $\Theta$ between two inflection points $(\times)$ in HGS MEA. $(D)$ Combination of yield strength $\left(\sigma_{0.2}\right)$ and uniform elongation $\left(E_{u}\right)$, in comparison with previous single-phase FCC MEAs and HEAs. Note the curvature of the $\sigma_{0.2}-\mathrm{E}_{u}$ relationship (red), which has a slope considerably higher than normally found. (Inset) The higher $\sigma_{0.2}$, the higher the advantage in increased $\mathrm{E}_{u}$.

annealing, as a representative of conventional alloys with uniform small grain size. The other is the $\mathrm{CG} \mathrm{CrCoNi}$ MEA after recrystallization annealing at $1,150{ }^{\circ} \mathrm{C}$ for $1 \mathrm{~h}$ (see details in $\mathrm{SI} \mathrm{Ap}$ pendix, Fig. S4). Obviously, the width of hysteresis loop in both cases is nowhere close to our HGS (see SI Appendix, Figs. S2F and $\mathrm{S} 4 D$ ). The larger the loop width (characterized by residual plastic strain, $\varepsilon_{r p}$, following elastic unloading), the stronger the back stress hardening $(47,52)$. In fact, the loop widths in the HGS MEA, as plotted in Fig. $3 B$, Inset, are by far the largest ever for any known alloy. Such actual experimental evidence is not available in the literature on HGSs, such as refs. 54-56, where it was only argued that such behavior may be possible. More directly, we observe that the increase in internal back stresses (see SI Appendix, Fig. S5 for the method of back stress calculations) is the decisive contributor to flow stress after yielding (Fig. 3C), accounting for over $80 \%$ of the total stress elevation observed during strain hardening, from yield stress to ultimate tensile stress. We thus conclude that back stress hardening takes a leading role in strain hardening accompanying the tensile elongation. The microstructural origin of the large back stress is the dynamically reinforced heterogeneous structure, to be further elaborated below.

The HGS MEA is, in fact, further refined and reinforced throughout the tensile deformation. To see this, we note that the number density of NGs ( $\rho_{\mathrm{NG}}$, grains per square micrometer) significantly increases from 4.7 grains per square micrometer (before tensile test in Fig. $1 A$ ) to 21 grains per square micrometer (after test at $298 \mathrm{~K}$ in Fig. $4 A$ ), and up to 31 grains per square micrometer (at $77 \mathrm{~K}$ in Fig. $4 B$ ) by statistical analysis of EBSD observations. The evolution of $\rho_{\mathrm{NG}}$ with tensile strain is further shown in Fig. $4 C$. The corresponding area fraction of NGs rises from $2.0 \%$ (before), to $5.5 \%(298 \mathrm{~K})$, and to $8.0 \%(77 \mathrm{~K})$. Correspondingly, the average grain size of NGs decreases from $180 \mathrm{~nm}$ (before) to $144 \mathrm{~nm}$ (tested at $298 \mathrm{~K}$ ), and further down to
$140 \mathrm{~nm}(77 \mathrm{~K})$, as seen in Fig. $4 C$. This clearly indicates the dynamic generation of smaller NGs during tensile deformation. EBSD statistical analysis indicates that the areas of UFGs and MGs decrease, with their number densities largely unchanged, as they provide the locations where NGs form.

The NGs show several interesting features. First, almost all NGs nucleate at the GBs where stresses are higher especially at GBs of UFGs (Fig. $4 A$ and $B$, Insets). Second, in terms of statistical EBSD analysis as shown in Fig. $4 C, \rho$ of corner twins (with $60^{\circ} \mathrm{C}$ misorientation in Fig. $4 D$ ) increases from 3.4 grains per square micrometer (before) to 8.0 grains per square micrometer $(298 \mathrm{~K})$ and up to 13.6 grains per square micrometer $(77 \mathrm{~K})$, respectively, although the TBs evolve with tensile straining and gradually lose the $\Sigma 3$ character as more and more SFs form inside the twinned grains (42). Third, $\rho_{\mathrm{NG}}$ begins to increase at a strain of $5 \%$ during tensile deformation at $298 \mathrm{~K}$, consistent with that where the upturn of $\Theta$ occurs (Fig. 2C). Namely, the generation of NGs corresponds to the upturn of $\Theta$. This is because stresses are high at HAGBs of UFGs as a result of load partitioning during tensile straining, facilitating the evolution from $\Sigma 3$ twin boundaries to HAGBs. With further increased strains, both the corner twins and NGs increase their $\rho$ in the later stage of tensile strains (Fig. 4C). In other words, the larger (MG) grains would carry more and more plastic strain as tensile deformation continues. At larger tensile strains, stress partitioning toward UFGs has increased the driving stress to sufficiently high levels for twin nucleation at the GBs of UFGs. New twinned NGs are therefore generated, through the emission of twinning partial dislocations (42) at these high-stress GB sites, increasing quickly with strain (Fig. 4D). The GBs of these NGs are effective high-angle barriers that impede the slip of dislocations. This refinement also makes the grain structure even more heterogeneous, perpetuating back stress hardening during subsequent deformation. 

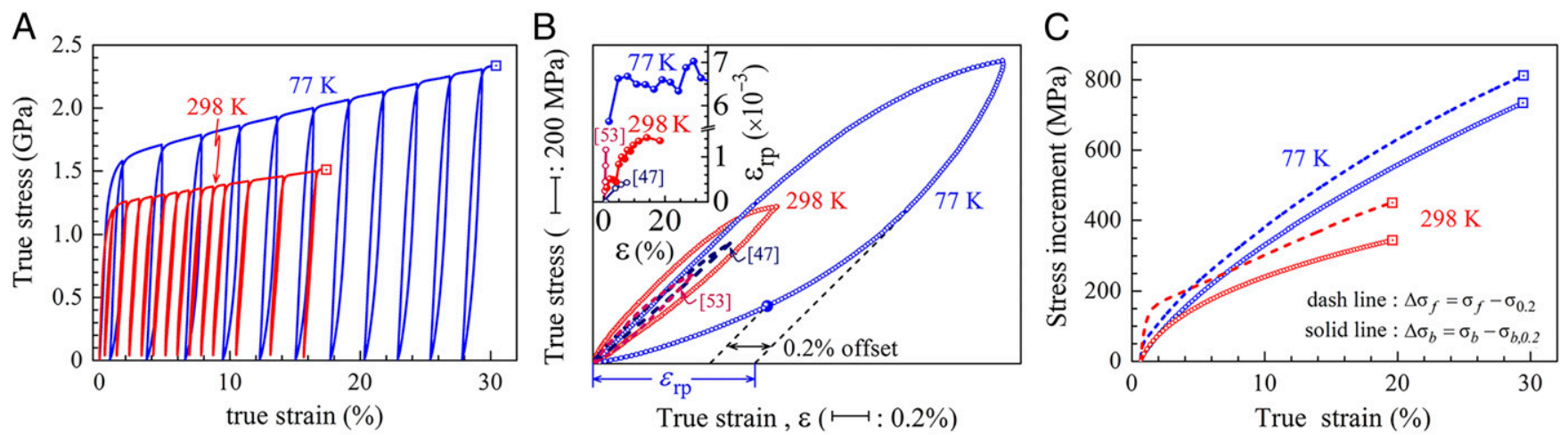

Fig. 3. Extraordinary back stress hardening in HGS. (A) Tensile true stress-true strain curves during LUR tests at 298 and $77 \mathrm{~K}$. (B) Hysteresis loops at the maximum uniform strain. Note inverse (compressive) yielding at $0.2 \%$ offset strain following elastic unloading even when the applied stress state is tensile. Hysteresis loops from other representative materials $(47,53)$ are also included for comparison. Also see comparison with narrow loops observed for both the UFG Ni in SI Appendix, Fig. S2F and coarse-grained CrCoNi MEA in SI Appendix, Fig. S4E. Inset shows the reverse plastic strain ( $\varepsilon_{\mathrm{rp}}$ ) versus applied strain. (C) Comparison of back stress hardening with the total strain hardening (measured flow stress minus that at yielding) in HGS MEA tested at 298 and $77 \mathrm{~K}$.

The dynamic generation of NGs in our HGS is further confirmed by TEM observations. SI Appendix, Fig. S6 shows clearly that the NGs indeed form during tensile straining, by monitoring and comparing the microstructural evolution after the cold rolling, recrystallization annealing, and tensile deformation. After tensile deformation, an original grain is often subdivided, due to the formation of NGs along the GB (Fig. $4 E$ ). SFs are also visible in most grains (Fig. $4 E$, Inset). Fig. $4 F$ shows several smaller NGs (green) that form at the GB of a parent UFG (yellow) during tensile deformation. They show twinning relationship with respect to the parent grain. Note here that it is the partially recrystallized HGS, in lieu of uniform-sized grains, that allows the new (twinned) NGs to emerge in large numbers on the fly, with associated strain hardening. A fully recrystallized grain structure would primarily store SFs, and would not be as conducive to load partitioning and dynamic nucleation of many (twinned) NGs at GBs and TJs via dynamic recrystallization. Also note that the high efficacy of dynamic refinement is also due to the low SFE, which facilitates the formation of corner and necklace twins that evolve into new NGs.

We next explain the evolution of $\Theta$ during tensile deformation, from the standpoint of the beneficial effect of the HGS on instigating the high internal back stress. Compared with uniform grains, the HGS adds an advantage in that plastic deformation is nonhomogeneous due to the inhomogeneous microstructure, inducing strain gradient and large internal stresses (46, 57-59). In contrast to the typical monotonic drop in $\Theta$ in the uniformly grained structures (curves 1 to 5 in Fig. 2C), the decreasing $\Theta$ of the HGS shows an upturn, typical of discontinuous yielding in elastoplastic transition, which, in the HGS, encompasses "grain to grain yielding": Upon tensile straining, the softer grains begin to yield first. Due to the constraints by the still-elastic small grains, dislocations in larger grains are piled up and blocked at grain boundaries. The
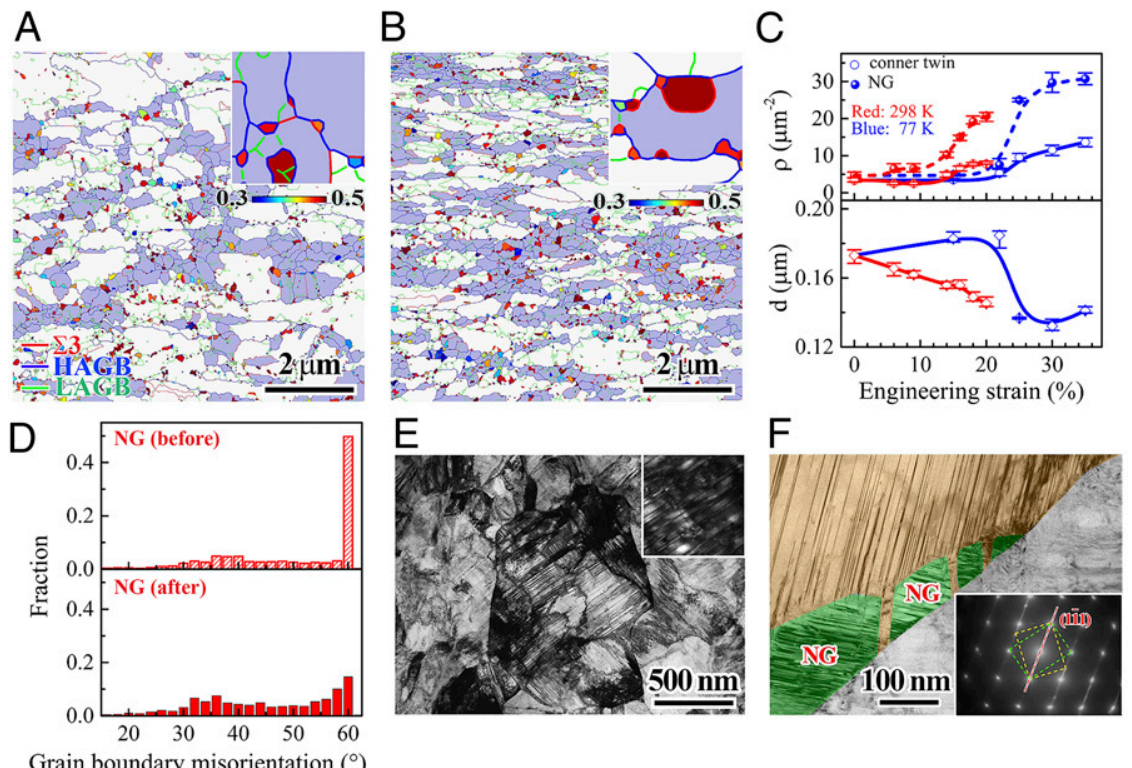

Grain boundary misorientation $\left(^{\circ}\right)$

Fig. 4. Dynamic generation of nanograins at GBs during tensile straining. ( $A$ and $B)$ EBSD grain boundary maps after tensile test at ( $A) 298$ and $(B) 77$ $\mathrm{K}$, respectively. (Insets) The generation of NGs mainly at GBs of UFGs. (Scale bar: magnitude of the Schmid factor.) (C) (Upper) Evolution of number density $(\rho)$ of both NGs and corner twins in tensile tests at 298 and $77 \mathrm{~K}$. (Lower) Grain size change of NGs during tensile deformation. (D) Distribution of grain boundary orientation in NGs before and after tensile test at $298 \mathrm{~K}$. (E) TEM image showing the dynamically generated NGs at $298 \mathrm{~K}$. (Inset) SFs of high density. $(F)$ TEM image showing the formation of twinned NGs at GB at $298 \mathrm{~K}$. (Inset) The diffraction pattern showing twinning orientation relationship between the twinned NG and parent grain. 
excess geometrically necessary dislocations (GNDs) will be generated due to strain incompatibility, to accommodate the strain gradient $(46,54,57-59)$. These GNDs may interact with mobile dislocations to increase the density of mobile dislocations in varying grains due to dislocation interaction and entanglement. Meanwhile, the MGs preferentially carry plastic strain, relative to the stronger small grains. This is accompanied by stress partitioning: The UFGs/NGs bear an increasing fraction of the applied stress, while MGs carry proportionally less stress. This generates back stresses, contributing to the back stress-induced hardening during the tensile deformation (47, 48, 52-54); see Fig. 3C. In other words, back stress hardening is associated with strain partitioning, i.e., nonhomogeneous plastic strain: A steep strain gradient is established at the grain boundary between grains of very different sizes $(47,57)$. Due to the constraints by the still-elastic small grains, dislocations in larger grains are piled up and blocked at grain boundaries. The long-range back stresses are significant as a result (Fig. 3 ), in addition to the hardening from forest dislocations in the grains, such that $\Theta$ exhibits an upturn; see Fig. $2 C$.

One note to add is that the deformation-generated NGs are themselves deformable and hardenable, via the many SFs generated inside them (see TEM image in Fig. $4 E$ and $F$ ). However, our emphasis here is that the dynamically generated NGs promote a flow response analogous to that in a multiphase (such as steels) or composite alloy. Specifically, the HGS is dynamically maintained and always plastically nonhomogeneous (when the overall sample is undergoing homogeneous plastic deformation). As demonstrated by the unprecedented magnitude of the long-range back stresses shown in Fig. $3 B$ and $C$, the strain inhomogeneity in the selfreinforcing HGS creates unusually large inhomogeneous internal stresses that promote extra hardening $(47,48,53,57,58)$. The strain hardening rate is therefore sustained (Fig. $2 C$ ) to prolong uniform elongation to large strains.

Finally, the strength-ductility combination offered by the HGS is much better than those reported before for all of the other single-phase FCC HEAs/MEAs (Fig. 2D) and TWIP steels with homogeneous microstructure (SI Appendix, Fig. S3A): We now have extended uniform ductility to $22 \%$ even when yield strength is pushed to above $1 \mathrm{GPa}$. The previous strength-ductility synergy was reported only for those with yield strength of $<400 \mathrm{MPa}$ (Fig. 2D), where the strain hardening capability is much easier to come by, as the room for defect storage has not been exhausted. There are cases, such as duplex steels or TWIP steels with $\mathrm{V}$ and $\mathrm{Cr}$ additions $(32,37)$, which have strength-ductility comparable to our HGS, as shown in SI Appendix, Fig. S3B. However, these enhanced properties are obtained for alloys containing more than one phase as heterogeneities. In this case, multiple mechanisms operate simultaneously for work hardening, including the TRIP effect, precipitates [vanadium carbide (VC) nanoprecipitation at $\mathrm{GBs}, \mathrm{Mo}_{2} \mathrm{C}$, etc.], TWIP, dislocations, etc. In particular, with complex composition (C, Mn, Al, Mo, and V, etc.), dual phases form to provide heterogeneity. In contrast, in MEAs/HEAs (Fig. 2D) and TWIP steels (SI Appendix, Fig. S3A), only one FCC phase is present, with no TRIP and precipitates in action. In other words, our HGS shows that a single-phase FCC alloy can now be made with a multilevel grain structure heterogeneity to reach properties achievable in a multi(dual) phase heterogeneous steel.

In summary, we advocate a strategy to achieve simultaneous high yield strength and high tensile ductility, generally applicable to low SFE metals and alloys, for which the CrCoNi MEA alloy serves as a model system. The key ingredient is to start out with a gigapascal yield strength from a purposely and extraordinarily heterogeneous grain structure that spans the nano-to-micro range. The HGS imparts an additional long-range hardening effect from back stresses, and is dynamically reinforced through the in situ production of new nanograins at GBs throughout tensile straining. In other words, the partially recrystallized starting grain structure facilitates nonhomogeneous plastic deformation and the associated strain gradient hardening. Also importantly, the HGS evolves toward an even more heterogeneous structure during tensile deformation, as more twins, faults, and NGs with high-angle GBs form on the fly due to the low SFE, rather than just dislocations that annihilate easily. This preserves a steep strain gradient across neighboring grains and hence large internal back stresses (see wide hysteresis loops) to sustain strain hardening over a range of plastic strains. We also showed that these effects would diminish in MEAs after extended annealing toward uniform grains, or in materials with high SFE where full dislocations dominate and could not accumulate efficiently due to recovery and annihilation into GBs. The dynamic HGS supplements the well-known effects of phase transformation (TRIP) (10) and twinning (TWIP) $(1,9,51)$, and are particularly useful for boosting strain hardening and uniform tensile ductility in low-SFE materials with high yield strength.

\section{Materials and Methods}

Material Fabrication and Sample Preparation. In the present study, an equimolar CrCoNi MEA was fabricated. The purity of each raw elemental material was higher than 99.9 wt \%. A bulk ingot, weighting $\sim 3 \mathrm{~kg}$, was produced by electromagnetic levitation melting in a high-purity argon atmosphere and cast to an ingot with a diameter of $120 \mathrm{~mm}$ and a height of $100 \mathrm{~mm}$. The ingot was then remelted five times to improve the homogeneity of both the chemical composition and microstructure. The actual chemical composition was measured to be $30.77 \mathrm{Cr}-33.81 \mathrm{Co}-35.34 \mathrm{Ni}$ (wt \%), i.e., $33.48 \mathrm{Cr}-32.46 \mathrm{Co}-34.06 \mathrm{Ni}$ (atomic percent). The ingot was homogenized at 1,473 $\mathrm{K}$ for $12 \mathrm{~h}$, hotforged in between $1,323 \mathrm{~K}$ and $1,173 \mathrm{~K}$ into slabs of $10 \mathrm{~mm}$ in thickness, and finally cold rolled into sheets $0.5 \mathrm{~mm}$ thick. No cracks were observed on the surfaces of the cold-rolled sheets. The sheets were subsequently annealed at varying temperatures ranging from $473 \mathrm{~K}$ to $1,473 \mathrm{~K}$ for $1 \mathrm{~h}$ followed by water quenching. The data presented in this paper are for annealing at $873 \mathrm{~K}$ for $1 \mathrm{~h}$, which produced a partially recrystallized microstructure.

Mechanical Property Testing. The flat dog-bone-shaped tensile specimens, with a gauge length of $15 \mathrm{~mm}$ and width of $4 \mathrm{~mm}$, were cut from the annealed sheets with their longitudinal axes parallel to the rolling direction. All specimens were mechanically polished before tensile tests to remove surface irregularities and to ensure a more accurate determination of the cross-sectional area. The quasi-static uniaxial tensile tests were carried out using an MTS Landmark testing machine operating at a strain rate of $5 \times$ $10^{-4} \mathrm{~s}^{-1}$. The tensile tests were conducted at room temperature $(298 \mathrm{~K})$ and liquid nitrogen temperature $(77 \mathrm{~K})$, respectively. The LUR tensile tests were conducted to characterize the back stresses. The methods for calculating the back stresses from LUR tensile test curves can be found in a previous paper (48). The conditions for LUR tests were the same as those for monotonic tests. All tensile tests were conducted using a $10-\mathrm{mm}$-gauge extensometer to monitor the engineering strain. Five samples were tested for each condition to verify the reproducibility.

Microstructural Characterization. SEM (JEOL JSM-7001F) was used to characterize the cross-sectional microstructural evolution of the annealed $\mathrm{CrCoNi}$ MEA before and after tensile tests. EBSD measurements were performed with a DigiView camera and the TSL OIM data collection software (www.edax. com/Products/EBSD/OIM-Data-Collection-EBSD-SEM.aspx), at an EBSD step size of $40 \mathrm{~nm}$. The grain boundary was defined by misorientation angle larger than $15^{\circ}$. The nanograins were colored based on the magnitude of the Schmid factor (60), in the undeformed and deformed microstructure, to partially reflect the orientation change, and, in turn, the deformation level, of nanograins during tensile deformation. Local grain misorientation information is provided through the grain reference orientation distribution (61) in SI Appendix, Fig. S7, to help track recrystallized grains in the HGS.

A high-spatial-resolution analytical electron microscope (JEM 2010F) operating at $200 \mathrm{kV}$ was used for examination of the microstructural features after tensile testing at designated strains. Thin foils for TEM observations were cut from the gauge sections of the tensile samples, ion-thinned to about $40 \mu \mathrm{m}$ thick, and finally thinned by a twin-jet polishing facility using a solution of $5 \%$ perchloric acid and $95 \%$ ethanol at $-25^{\circ} \mathrm{C}$.

ACKNOWLEDGMENTS. X.W. and F.Y. are funded by the National Key R\&D Program of China (Grant 2017YFA0204402), the Strategic Priority Research Program of the Chinese Academy of Sciences (Grant XDB22040503), and the Natural Science Foundation of China (Grants 11572328, 11790293, and 11472286). E.M. was supported by US Department of Energy, Division of Materials Sciences and Engineering, under Contract DE-FG02-16ER46056. 
1. Gludovatz B, et al. (2016) Exceptional damage-tolerance of a medium-entropy alloy CrCoNi at cryogenic temperatures. Nat Commun 7:10602.

2. Zhang Z, et al. (2017) Dislocation mechanisms and 3D twin architectures generate exceptional strength-ductility-toughness combination in $\mathrm{CrCoNi}$ medium-entropy alloy. Nat Commun 8:14390.

3. Yoshida S, Bhattacharjee T, Bai Y, Tsuji N (2017) Friction stress and Hall-Petch relationship in $\mathrm{CoCrNi}$ equi-atomic medium entropy alloy processed by severe plastic deformation and subsequent annealing. Scr Mater 134:33-36.

4. Wu Z, Bei H, Pharr GM, George EP (2014) Temperature dependence of the mechanical properties of equiatomic solid solution alloys with face-centered cubic crystal structures. Acta Mater 81:428-441.

5. Laplanche G, et al. (2017) Reasons for the superior mechanical properties of mediumentropy CrCoNi compared to high-entropy CrMnFeCoNi. Acta Mater 128:292-303.

6. Miao J, et al. (2017) The evolution of the deformation substructure in a Ni-Co-Cr equiatomic solid solution alloy. Acta Mater 132:35-48.

7. Shahmir H, He JY, Lu ZP, Kawasaki M, Langdon TG (2016) Effect of annealing on mechanical properties of a nanocrystalline CoCrFeNiMn high-entropy alloy processed by high-pressure torsion. Mater Sci Eng A 676:294-303.

8. Yeh JW, et al. (2004) Nanostructured high-entropy alloys with multiple principal elements: Novel alloy design concepts and outcomes. Adv Eng Mater 6:299-303.

9. Gludovatz B, et al. (2014) A fracture-resistant high-entropy alloy for cryogenic ap plications. Science 345:1153-1158.

10. Li Z, Pradeep KG, Deng Y, Raabe D, Tasan CC (2016) Metastable high-entropy dualphase alloys overcome the strength-ductility trade-off. Nature 534:227-230.

11. Zou Y, Ma H, Spolenak R (2015) Ultrastrong ductile and stable high-entropy alloys at small scales. Nat Commun 6:7748.

12. Zhang Z, et al. (2015) Nanoscale origins of the damage tolerance of the high-entropy alloy CrMnFeCoNi. Nat Commun 6:10143.

13. Chen BR, Yeh AC, Yeh JW (2016) Effect of one-step recrystallization on the grain boundary evolution of CoCrFeMnNi high entropy alloy and its subsystems. Sci Rep 6 : 22306.

14. He JY, et al. (2016) A precipitation-hardened high-entropy alloy with outstanding tensile properties. Acta Mater 102:187-196.

15. Li DY, et al. (2017) High-entropy $\mathrm{Al}_{0.3} \mathrm{CoCrFeNi}$ alloy fibers with high tensile strength and ductility at ambient and cryogenic temperatures. Acta Mater 123:285-294.

16. $\mathrm{Ma} Y$, et al. (2018) Dynamic shear deformation of a crconi medium-entropy alloy with heterogeneous grain structures. Acta Mater 148:407-418.

17. Zaddach AJ, Scattergood RO, Koch CC (2015) Tensile properties of low-stacking fault energy high-entropy alloys. Mater Sci Eng A 636:373-378.

18. Wu Z, Gao Y, Bei H (2016) Thermal activation mechanisms and Labusch-type strengthening analysis for a family of high-entropy and equiatomic solid-solution alloys. Acta Mater 120:108-119.

19. Stepanov N, et al. (2015) Effect of cryo-deformation on structure and properties of CoCrFeNiMn high-entropy alloy. Intermetallics 59:8-17.

20. Schuh B, et al. (2015) Mechanical properties, microstructure and thermal stability of a nanocrystalline CoCrFeMnNi high-entropy alloy after severe plastic deformation. Acta Mater 96:258-268.

21. Salishchev GA, et al. (2014) Effect of Mn and V on structure and mechanical propertie of high-entropy alloys based on CoCrFeNi system. J Alloys Compd 591:11-21.

22. Li ZM, Tasan CC, Pradeep KG, Raabe D (2017) A TRIP-assisted dual-phase high-entropy alloy: Grain size and phase fraction effects on deformation behavior. Acta Mater 131 323-335.

23. Li ZM, Körmann K, Grabowski B, Neugebauer J, Raabe D (2017) Ab initio assisted design of quinary dual-phase high-entropy alloys with transformation-induced plasticity. Acta Mater 136:262-270.

24. Wang MM, Li ZM, Raabe D (2018) In-situ SEM observation of phase transformation and twinning mechanisms in an interstitial high-entropy alloy. Acta Mater 147: 236-246.

25. Smith TM, et al. (2016) Atomic-scale characterization and modeling of 60 degrees dislocations in a high-entropy alloy. Acta Mater 110:352-363.

26. Meyers MA, Chawla KK (2009) Mechanical Behavior of Materials (Cambridge Univ Press, Cambridge, UK)

27. Wang Y, Chen M, Zhou F, Ma E (2002) High tensile ductility in a nanostructured metal. Nature 419:912-915.

28. Lu L, Shen Y, Chen X, Qian L, Lu K (2004) Ultrahigh strength and high electrical conductivity in copper. Science 304:422-426.

29. Zhu YT, Liao X (2004) Nanostructured metals: Retaining ductility. Nat Mater 3: 351-352.

30. Grassel O, Kruger L, Frommeyer G, Meyer LW (2000) High strength Fe-Mn-(AI, Si) TRIP/ TWIP steels development-properties-application. Int J Plast 16:1391-1409.

31. Wu XL, Yang MX, Yuan FP, Chen L, Zhu YT (2016) Combining gradient structure and TRIP effect to produce austenite stainless steel with high strength and ductility. Acta Mater 112:337-346.
32. Sohn SS, et al. (2017) Novel $1.5 \mathrm{GPa}$-strength with $50 \%$-ductility by transformationinduced plasticity of non-recrystallized austenite in duplex steels. Sci Rep 7:1255.

33. Herrera C, Ponge D, Raabe D (2011) Design of a novel Mn-based 1 GPa duplex stainless TRIP steel with $60 \%$ ductility by a reduction of austenite stability. Acta Mater 59:4653-4664.

34. Chen XH, Lu J, Lu L, Lu K (2005) Tensile properties of a nanocrystalline 316 L austenitic stainless steel. Scr Mater 52:1039-1044.

35. Chen AY, Li DF, Zhang JB, Song HW, Lu J (2008) Make nanostructured metal exceptionally tough by introducing non-localized fracture behaviors. Scr Mater 59:579-582.

36. Dini G, Najafizadeh A, Ueji R, Monir-Vaghefi SM (2010) Improved tensile properties of partially recrystallized submicron grained TWIP steel. Mater Lett 64:15-18.

37. Niendorf T, Rüsing CJ, Frehn A, Maier HJ (2013) The deformation behavior of functionally graded TWIP steel under monotonic loading at ambient temperature. Mater Res Lett 1:96-101.

38. Sohn SS, Song H, Kwak JH, Lee S (2017) Dramatic improvement of strain hardening and ductility to $95 \%$ in highly-deformable high-strength duplex lightweight steels. Sci Rep 7:1927.

39. Chen S, Rana R, Haldar A, Ray RK (2017) Current state of Fe-Mn-Al-C low density steels. Prog Mater Sci 89:345-391.

40. Wang MM, Tasan CC, Ponge D, Dippel AC, Raabe D (2015) Nanolaminate transformation-induced plasticity/twinning-induced plasticity steel with dynamic strain partitioning and enhanced damage resistance. Acta Mater 85:216-228.

41. Gutierrez-Urrutia I, Raabe D (2011) Dislocation and twin substructure evolution during strain hardening of an Fe-22wt.\% Mn-0.6wt.\% C TWIP steel observed by electron channeling contrast imaging. Acta Mater 59:6449-6462.

42. Zhu YT, Liao XZ, Wu XL (2012) Deformation twinning in nanocrystalline materials. Prog Mater Sci 57:1-62.

43. Wu XL, Zhu YT (2008) Inverse grain-size effect on twinning in nanocrystalline Ni. Phys Rev Lett 101:025503.

44. Sakai T, Belyakov A, Kaibyshev R, Miura H, Jonas JJ (2014) Dynamic and post-dynamic recrystallization under hot, cold and severe plastic deformation conditions. Prog Mater Sci 60:130-207.

45. Ponge D, Gottstein G (1998) Necklace formation during dynamic recrystallization: Mechanisms and impact on flow behavior. Acta Mater 46:69-80.

46. Wu XL, Jiang P, Chen L, Yuan FP, Zhu YT (2014) Extraordinary strain hardening by gradient structure. Proc Natl Acad Sci USA 111:7197-7201.

47. $\mathrm{Wu} \mathrm{XL}$, et al. (2015) Heterogeneous lamella structure unites ultrafine-grain strength with coarse-grain ductility. Proc Natl Acad Sci USA 112:14501-14505.

48. Yang MX, Pan Y, Yuan FP, Zhu YT, Wu XL (2016) Back stress strengthening and strain hardening in gradient structure. Mater Res Lett 4:145-151.

49. Wu XL, et al. (2014) Synergetic strengthening by gradient structure. Mater Res Lett 2: 185-191.

50. Odnobokova M, Belyakov A, Kaibyshev R (2015) Effect of severe cold or warm deformation on microstructure evolution and tensile behavior of a $316 \mathrm{~L}$ stainless steel. Adv Eng Mater 17:1812-1820.

51. Li Z, Tasan CC, Springer H, Gault B, Raabe D (2017) Interstitial atoms enable joint twinning and transformation induced plasticity in strong and ductile high-entropy alloys. Sci Rep 7:40704.

52. Sinclair CW, Saada G, Embury JD (2006) Role of internal stresses in co-deformed twophase materials. Philos Mag 86:4081-4098.

53. Xiang Y, Vlassak JJ (2006) Bauschinger and size effects in thin-film plasticity. Acta Mater 54:5449-5460.

54. Wu XL, Zhu YT (2017) Heterogeneous materials: A new class of materials with unprecedented mechanical properties. Mater Res Lett 5:527-532.

55. Liu XL, Yuan FP, Zhu YT, Wu XL (2018) Extraordinary Bauschinger effect in gradient structured copper. Scr Mater 150:57-60.

56. Ma E, Zhu T (2017) Towards strength-ductility synergy through the design of heterogeneous nanostructures in metals. Mater Today 20:323-331.

57. Ashby MF (1970) The deformation of plastically non-homogeneous materials. Philos Mag 21:399-424

58. Gao H, Huang Y, Nix WD, Hutchinson JW (1999) Mechanism-based strain gradient plasticity-I. Theory. J Mech Phys Solids 47:1239-1263.

59. Huang CX, et al. (April 2018) Interface affected zone for optimal strength and ductility in heterogeneous laminate. Mater Today, 10.1016/j.mattod.2018.03.006.

60. Blochwitz C, Brechbuehl J, Tirschler W (1996) Analysis of activated slip systems in fatigued nickel polycrystals using the EBSD-technique in the scanning electron microscope. Mater Sci Eng A 210:42-47.

61. Gutierrez-Urrutia I, et al. (2016) Plastic accommodation at homophase interfaces between nanotwinned and recrystallized grains in an austenitic duplex-microstructured steel. Sci Technol Adv Mater 17:29-36. 